\title{
SOUSLIN DENDRONS
}

\author{
J. van MILL AND E. WATTEL
}

\begin{abstract}
A dendron is a continuum in which every two distinct points have a separation point. We call a dendron $X$ a Souslin dendron provided that $X$ satisfies the countable chain condition, is not separable and has the additional property that every countable subset of $X$ is contained in a metrizable subcontinuum of $X$. We prove that the existence of a Souslin line is equivalent to the existence of a Souslin dendron. In addition, each Souslin dendron is a continuous image of some Souslin continuum.
\end{abstract}

1. Introduction. A dendron or compact tree-like space is a connected compact Hausdorff space (or briefly "continuum") in which every two distinct points have a separation point. Clearly every orderable continuum is a dendron; however the class of dendrons is much bigger (see e.g. Kok [7]).

We call a dendron $X$ a Souslin dendron provided that it satisfies the following three conditions

(i) $X$ satisfies the countable chain condition;

(ii) $X$ is not separable;

(iii) each countable subset of $X$ is contained in a metrizable subcontinuum of $X$.

(Notice that the condition (iii) implies that a Souslin line never is a Souslin dendron.)

In this paper we will prove that the existence of a Souslin line is equivalent to the existence of a Souslin dendron. Hence $\diamond$ implies that there is a Souslin dendron (cf. Jensen [5]) and MA $+\neg \mathrm{CH}$ implies that there is no Souslin dendron (cf. Rudin [13]). We will also prove that each Souslin dendron is the continuous image of some Souslin line.

2. Dendrons. Let $X$ be a dendron. For all distinct $a, b \in X$ let $S(a, b) \subset X$ be defined by

$$
S(a, b):=\{x \in X \mid x \text { separates } a \text { from } b\} \cup\{a, b\} .
$$

It is well known that $S(a, b)$ is an orderable continuum (cf. Proizvolov [10], Kok [7]). In fact $S(a, b)$ is ordered by the usual cut point ordering. In [7], Kok has proved that $S(a, b)$ can also be represented as the intersection of all closed connected subsets of $X$ containing $\{a, b\}$. This implies that if $X$ is a Souslin dendron then $S(a, b)$ is metrizable and moreover $S(a, b)$ is order isomorphic to $[0,1]$, the closed unit interval (cf. Ward [14]). This observation leads to the following:

Received by the editors October 24, 1977 and, in revised form, February 22, 1978.

AMS (MOS) subject classifications (1970). Primary 54F50, 54F05.

Key words and phrases. Souslin line, dendron, inverse limit, monotone mapping. 
2.1 Lemma. Let $X$ be a dendron which is ccc and not separable. Then the following statements are equivalent:

(i) $X$ is a Souslin dendron;

(ii) for all distinct $a, b \in X: S(a, b)$ is metrizable;

(iii) for all distinct $a, b \in X: S(a, b)$ is homeomorphic to $[0,1]$;

(iv) for all distinct $a, b \in X: S(a, b)$ is separable.

Proof. (iv) $\Rightarrow$ (i) The union of all $S\left(a_{1}, a_{2}\right)$ with $a_{1}, a_{2} \in A$, in which $A$ is a countable subset of $X$, is separable. Its closure is contained in a separable dendron, and (i) now follows from Proizvolov [11]. The other implications are clear.

Let $X$ be a dendron. For all $B \subset X$ the intersection of all subcontinua of $X$ containing $B$ is denoted by $S(B)$. By the above cited result of Kok [7] it follows that $S(B)$ is a subcontinuum of $X$.

The following proposition follows from earlier results (cf. van Mill and Schrijver [8], van Mill and van de Vel [9]).

2.2 Proposition. Let $X$ be a dendron and let $A \subset X$ be a subcontinuum. Then the mapping $r: X \rightarrow A$ defined by

$$
\{r(x)\}=\bigcap_{a \in A} S(\{x, a\}) \cap A
$$

is a retraction.

3. ccc Dendrons. In this section we investigate some special properties of ccc dendrons and prove an important lemma which is used in the proof of the main result in the present paper.

3.1 ThEOREM. Let $X$ be a dendron which satisfies the countable chain condition. Then $X$ is hereditarily ccc, hereditarily Lindelöf and consequently is perfectly normal.

Proof. By a result of Cornette [2] there is an ordered continuum $L$ and a continuous surjection $f: L \rightarrow X$. Let $A \subset L$ be a closed set such that $f \uparrow A$ is irreducible, that is, if $B$ is a closed subset of $A$ with $f[B]=X$ then $B=A$ (the existence of $A$ is an easy consequence of Zorn's lemma). For all nonvoid open $U \subset A$ define $U^{\sharp} \subset X$ by $U^{\sharp}:=X \backslash f[A \backslash U]$. Since $f$ is closed and irreducible the set $U^{\sharp}$ is open and nonvoid. In addition, for all nonvoid open $U, V \subset A$ we have that $U \cap V=\varnothing$ implies $U^{\sharp} \cap V^{\sharp}=\varnothing$. This implies that $A$ is ccc. An ordered space which satisfies the countable chain condition is hereditarily ccc and hereditarily Lindelöf (this is well known, see for instance Faber [3]). We conclude that $X$ is hereditarily ccc and hereditarily Lindelöf.

3.2 Corollary (MA $+\neg \mathrm{CH})$. Let $X$ be a dendron. Then the following statements are equivalent:

(i) $X$ is metrizable; 
(ii) $X$ is ccc.

Proof. (i) $\Rightarrow$ (ii) is trivial. (ii) $\Rightarrow$ (i). Follows from $3.1 ; 2.1$ and Juhász [6].

3.3 LEMMA. Let $X$ be a dendron which satisfies the countable chain condition. Let $X_{\alpha}$ and $X_{\beta}$ be subcontinua of $X$ such that $X_{\alpha} \subset X_{\beta}$. Let $r_{\alpha}$ (resp. $r_{\beta}$ ) be the retractions of $X$ onto $X_{\alpha}$ (resp. $X_{\beta}$ ) described in Proposition 2.2. For $\delta \in\{\alpha, \beta\}$ let $M_{\delta}:=\left\{x \in X_{\delta}|| r_{\delta}^{-1}(x) \mid \geqslant 2\right\}$. Then

(i) for all $x \in X: r_{\alpha} r_{\beta}(x)=r_{\alpha}(x)$;

(ii) if $\delta \in\{\alpha, \beta\}$ then $r_{\delta}^{-1}(x) \backslash\{x\}$ is open for all $x \in X_{\delta}$;

(iii) if $\delta \in\{\alpha, \beta\}$ then $\left|M_{\delta}\right| \leqslant \omega$.

Proof. (i) is trivial using the precise definition of $r_{\alpha}$ and $r_{\beta}$ and (iii) follows from (ii) since $X$ is ccc. To prove (ii), take $x \in M_{\alpha}$ and $y \in r_{\alpha}^{-1}(x) \backslash\{x\}$ and let $p$ be a separation point of $x$ and $y$. Since $S(y, x) \cap M_{\alpha}=\{x\}$, as can easily be seen, it follows that $S(p, x) \cap M_{\alpha}$ also equals $\{x\}$ (notice that $S(p, x) \subset S(y, x))$. Consequently $r_{\alpha}(p)=x$, i.e. $p \in r_{\alpha}^{-1}(x)$. Let $U$ be the component of $X \backslash\{p\}$ containing $y$. Then $U$ is open since $X$ is locally connected (cf. Gurin [4]).

We claim that $U \subset r_{\alpha}^{-1}(x)$. Indeed, take $q \in U$. Then

$$
S(q, p) \subset U \cup\{p\},
$$

since $U \cup\{p\}$ is connected and closed. In addition, $U \cup\{p\}$ does not intersect $X_{\alpha}$, since $p$ separates $x$ from $y$ and $x \in X_{\alpha}$ and $X_{\alpha}$ is connected. Therefore

$$
\begin{aligned}
S(q, x) \cap X_{\alpha} & \subset\left(S(q, p) \cup S(p, x) \cap X_{\alpha}\right) \\
& =\left(S(q, p) \cap X_{\alpha}\right) \cup\left(S(p, x) \cap X_{\alpha}\right) \\
& \subset\left((U \cup\{p\}) \cap X_{\alpha}\right) \cup\{x\}=\{x\},
\end{aligned}
$$

which implies that $r_{\alpha}(q)=x$. Hence $U \subset r_{\alpha}^{-1}\{x\}$ and consequently $r_{\alpha}^{-1}(x) \backslash\{x\}$ is open.

4. The existence of Souslin dendrons. In this section we prove that the existence of a Souslin line implies the existence of a Souslin dendron. We use inverse limit techniques. For more information concerning inverse limits we refer to Capel [1].

$\left(X_{\alpha}, f_{\alpha \beta}, \kappa\right)$, where $\kappa$ is an ordinal number, means that for all $\alpha<\kappa, X_{\alpha}$ is a topological space and that for all $\beta<\alpha<\kappa, f_{\alpha \beta}: X_{\alpha} \rightarrow X_{\beta}$ is continuous such that $\gamma<\beta<\alpha$ implies that $f_{\alpha \gamma}=f_{\beta \gamma} \circ f_{\alpha \beta}$.

4.1 LEMMA. Let $X$ be a compact connected ordered space of weight at most $\omega_{1}$. Then there is an inverse system $\left(I_{\alpha}, f_{\alpha \beta}, \omega_{1}\right)$ where each $I_{\alpha}$ is a copy of the closed unit interval and where each $f_{\alpha \beta}$ is monotone and onto such that inv $\lim \left(I_{\alpha}, f_{\alpha \beta}, \omega_{1}\right)$ is homeomorphic to $X$. In addition, the mappings $f_{\alpha \beta}$ can be chosen in such a way that they have at most one nondegenerate point inverse if $\alpha=\beta+1$. 
Proof. Let $D$ be a dense subset of $X$ of cardinality at most $\omega_{1}$. Let

$$
E:=\left\{\left(d_{0}, d_{1}\right) \mid d_{0}, d_{1} \in D \text { and } d_{0} \neq d_{1}\right\} .
$$

In addition, let $\Gamma$ be the collection of nonlimit ordinals in $\omega_{1}$. List $E$ as $\left\{\left(d_{0}^{\gamma}, d_{1}^{\gamma}\right) \mid \gamma \in \Gamma\right\}$. We will now inductively construct the inverse system approximating $X$. We will do it in such a way that for each $\alpha<\omega_{1}$ there is a monotone surjection $\Pi_{\alpha}: X \rightarrow I_{\alpha}$ such that for all $\alpha<\beta<\omega_{1}$ we have that $\Pi_{\alpha}=f_{\beta \alpha} \circ \Pi_{\beta}$ while in addition for each $\gamma \in \Gamma$ the points $\Pi_{\gamma}\left(d_{0}^{\gamma}\right)$ and $\Pi_{\gamma}\left(d_{1}^{\gamma}\right)$ are distinct.

Suppose that we have completed the construction for all $\alpha<\beta$. First suppose that $\beta=0$. Without loss of generality assume that $d_{0}^{0}<d_{1}^{0}$. It now is easy to construct a monotone Urysohn mapping $f: X \rightarrow I=[0,1]$ such that $f\left(d_{0}^{0}\right)=0$ and $f\left(d_{1}^{0}\right)=1$. Define $I_{0}:=I$ and $\Pi_{0}:=f$. If $\beta \neq 0$ we consider the case that $\beta$ is a limit ordinal first. Consider the inverse system $\left(I_{\alpha}, f_{\alpha \gamma}, \beta\right)$. Since for each $\gamma<\beta$ there is, by induction hypothesis, a monotone surjection $\Pi_{\gamma}: X \rightarrow I_{\gamma}$ such that for each $\delta<\gamma<\beta$ the diagram

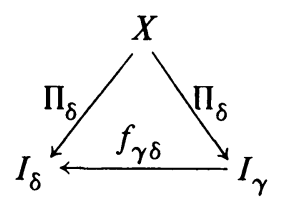

commutes, the mapping $e: X \rightarrow \operatorname{inv} \lim \left(I_{\alpha}, f_{\alpha \gamma}, \beta\right)$ defined by

$$
e(x)_{\gamma}:=\Pi_{\gamma}(x) \quad(\gamma<\beta)
$$

is a continuous surjection. It is easily seen that $e$ is monotone. Let $J=$ inv $\lim \left(I_{\alpha}, f_{\alpha \gamma}, \beta\right)$. By a result of Capel [1], $J$ is an ordered compactum, while in addition $J$ is metrizable since $\beta$ is a countable ordinal. Hence $J$ is homeomorphic to $[0,1]$. Define $I_{\beta}:=J$ and for all $\alpha<\beta$ let $f_{\beta \alpha}$ be the projection of $J=\operatorname{inv} \lim \left(I_{\alpha}, f_{\alpha \gamma}, \beta\right)$ onto $I_{\alpha}$; in addition define $\Pi_{\beta}:=e$. It is easy to show that our inductive assumptions are satisfied. If $\beta$ is a nonlimit, say $\beta=\alpha+1$, then there are two cases; if $\Pi_{\alpha}\left(d_{0}^{\beta}\right) \neq \Pi_{\alpha}\left(d_{1}^{\beta}\right)$ then we do nothing, i.e. we define $I_{\beta}:=I_{\alpha}, \Pi_{\beta}:=\Pi_{\alpha}$ and for all $\gamma<\beta$ define $f_{\beta \gamma}:=f_{\alpha \gamma}$ if $\gamma \neq \alpha$ and $f_{\beta \gamma}=\mathrm{id}_{X_{\alpha}}$ if $\gamma=\alpha$. Now suppose that $d_{0}^{\beta}<d_{1}^{\beta}$ and that $\Pi_{\alpha}\left(d_{0}^{\beta}\right)=\Pi_{\alpha}\left(d_{1}^{\beta}\right)$. (W.l.o.g. we may assume that $\Pi_{\alpha}$ is order preserving.)

Let $g:\left[d_{0}^{\beta}, d_{1}^{\beta}\right] \rightarrow I=[0,1]$ be a continuous monotone surjection such that $g\left(d_{0}^{\beta}\right)=0$ and $g\left(d_{1}^{\beta}\right)=1$. In $I_{\alpha}$ split the point $\Pi_{\alpha}\left(d_{0}^{\beta}\right)$ in two points, say $a$ and $b$; let $a<b$ and identify $a$ with 0 and $b$ with 1 . The resulting set $J$ is ordered in the natural way, hence is homeomorphic to the closed unit interval. Define a mapping $f: X \rightarrow J$ by

$$
\begin{cases}f(x)=\Pi_{\alpha}(x) & \text { if } x<a, \\ f(x)=\Pi_{\alpha}(x) & \text { if } b<x, \\ f(x)=g(x) & \text { if } a \leqslant b \leqslant x .\end{cases}
$$

Then $f$ is monotone and hence continuous. Let $h: J \rightarrow I_{\alpha}$ be the mapping 
which collapses $[a, b]$ to $\Pi_{\alpha}\left(d_{0}^{\beta}\right)$. Define $I_{\beta}:=J, \Pi_{\beta}:=f$ and $f_{\beta \alpha}:=h$. Finally define $f_{\beta \gamma}(\gamma<\alpha)$ as the composition of $f_{\beta \alpha}$ and $f_{\alpha \gamma}$. It is easily seen that our inductive hypotheses are satisfied. This completes the transfinite construction.

Now define a mapping $e: X \rightarrow \operatorname{inv} \lim \left(I_{\alpha}, f_{\alpha \beta}, \omega_{1}\right)$ by $e(x)_{\alpha}:=\Pi_{\alpha}(x)$. Then $e$ is well defined and consequently is a continuous surjection $(X$ is compact!). It suffices to prove that $e$ is one-to-one. Indeed, take $x, y \in X$ such that $x<y$; choose distinct $d_{0}, d_{1} \in D$ such that $x<d_{0}<d_{1}<y$. Then $\left(d_{0}, d_{1}\right) \in E$ and hence there is a nonlimit ordinal number $\beta$ such that $\Pi_{\beta}\left(d_{0}\right) \neq \Pi_{\beta}\left(d_{1}\right)$. By the fact that $\Pi_{\beta}$ is monotone it now follows that $\Pi_{\beta}(x) \neq \Pi_{\beta}(y)$; consequently $e(x) \neq e(y)$. We conclude that $e$ is a homeomorphism.

4.2 THEOREM. The existence of a Souslin line implies the existence of a Souslin dendron.

Proof. It is well known that the existence of a Souslin line implies the existence of a Souslin continuum of weight $\omega_{1}$, cf. Rudin [13]. Let $L$ be a Souslin continuum of weight (or, equivalently, density) $\omega_{1}$. By Lemma 4.1, $L \approx \operatorname{inv} \lim \left(I_{\alpha}, f_{\alpha \beta}, \omega_{1}\right)$ where each $f_{\alpha \beta}$ is monotone. For each $\alpha<\omega_{1}$ we construct a metric dendron $T_{\alpha}$ and a mapping $\xi_{\alpha}: I_{\alpha} \stackrel{\text { onto }}{\rightarrow} T_{\alpha}$ such that for each $\alpha<\beta<\omega_{1}$ there is a monotone retraction $r_{\beta \alpha}: T_{\beta} \rightarrow T_{\alpha}$ with the property that the diagram

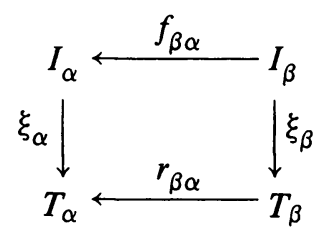

commutes.

Suppose that the construction is completed for all $\alpha<\beta<\omega_{1}$. If $\beta=0$ then define $T_{0}:=I_{0}$ and $\xi_{0}:=\mathrm{id}_{I_{0}}$. If $\beta$ is a limit ordinal set $T_{\beta}=$ inv $\lim \left(T_{\alpha}, r_{\alpha \gamma}, \beta\right)$ and define all mappings in the obvious way. It is easy to see that $T_{\beta}$ indeed is a dendron since the inverse limit of dendrons with monotone surjective bonding maps is a dendron (this result is not stated explicitly in Capel [1], but it can be proved using the same technique). If $\beta$ is a successor, say $\beta=\alpha+1$, consider the point $x$ for which $f_{\beta \alpha}^{-1}(x)$ is nondegenerate. (If it exists; otherwise we do nothing.)

Let $Z:=T_{\alpha} \times I$ and consider the subspace $Y=\left(T_{\alpha} \times\{0\}\right) \cup\left(\left\{\xi_{\alpha}(x)\right\} \times\right.$ I). This space clearly is a metric dendron and in addition the projection $\Pi_{1}$ : $Y \rightarrow T_{\alpha}$ onto the first coordinate is (equivalent to) a retraction. Let $a$ and $b$ be the endpoints of the interval $f_{\beta \alpha}^{-1}(x)$. Let $\phi: f_{\beta \alpha}^{-1}(x) \rightarrow I$ be a continuous surjection such that $\phi(a)=\phi(b)=0$. Now define $T_{\beta}:=Y, r_{\beta \alpha}:=\Pi_{1}$ and $\xi_{\beta}$ by the following rules 


$$
\begin{cases}\xi_{\beta}(y)=\left(\xi_{\alpha} f_{\beta \alpha}(y), 0\right) & \text { if } y \notin[a, b], \\ \xi_{\beta}(y)=\left(\xi_{\alpha}(x), \phi(y)\right) & \text { if } y \in[a, b] .\end{cases}
$$

It then is easily seen that our inductive hypotheses are satisfied.

Now put $T=\operatorname{inv} \lim \left(T_{\alpha}, r_{\alpha \beta}, \omega_{1}\right)$. Then there is a continuous surjection $f$ : $L \rightarrow T$ which implies that $T$ is perfectly normal and ccc. That $T$ is a dendron is obvious (cf. the above remark). Since each $r_{\beta \alpha}$ is a retraction it is easy to identify each $T_{\alpha}$ in a canonical way with a subspace of $T$. We will do so. Then since $T=\operatorname{inv} \lim \left(T_{\alpha}, r_{\alpha \beta}, \omega_{1}\right)$ we see that $\cup_{\alpha<\omega_{1}} T_{\alpha}$ is dense in $T$ and also that $\alpha<\beta$ implies that $T_{\alpha} \subset T_{\beta}$. Since $T$ is first countable (cf. 3.1) and since there are precisely $\omega_{1}$ distinct $T_{\alpha}$,s we conclude that $\cup_{\alpha<\omega_{1}} T_{\alpha}$ is closed in $T$. Consequently $\cup_{\alpha<\omega_{1}} T_{\alpha}=T$, since $\cup_{\alpha<\omega_{1}} T_{\alpha}$ is dense in $T$. This implies that $T$ is not separable and also that each countable subset of $T$ is contained in some $T_{\alpha}$. The $T_{\alpha}$ 's being metrizable we conclude that $T$ is a Souslin dendron.

5. The existence of Souslin lines. In this section we prove that each Souslin dendron is a continuous image of some Souslin line. As a corollary it follows that the existence of a Souslin line is equivalent to the existence of a Souslin dendron.

5.1 TheOREM. Each Souslin dendron is a continuous image of some Souslin continuum.

Proof. Let $T$ be a Souslin dendron. We construct a Souslin continuum $L$ which can be mapped continuously onto $T$ by means of transfinite induction using a suitable inverse limit system $\left(T_{\alpha}, r_{\alpha \beta}, \omega_{1}\right)$ of metrizable dendrons approximating $T$. The $T_{\alpha}$ 's are comparable subdendrons of $T$ and the $r_{\alpha \beta}$ 's are the canonical retractions between them (cf. Proposition 2.2).

For every $\alpha<\omega_{1}$ we now construct a metrizable subcontinuum $T_{\alpha} \subset T$ such that

(i) $\beta<\alpha<\omega_{1} \rightarrow T_{\beta} \subsetneq T_{\alpha}$;

(ii) $\left|r_{\alpha+1}^{-1}(x)\right|=1$ for each $x \in T_{\alpha}$, where $r_{\alpha+1}: T \rightarrow T_{\alpha+1}$ is the canonical retraction of $T$ onto $T_{\alpha+1}$.

Assume that we have completed the construction for all $\beta<\alpha<\omega_{1}$. If $\alpha=0$ then choose two distinct endpoints $a_{0}$ and $b_{0}$ of $T$ and define $T_{0}:=$ $S\left(a_{0}, b_{0}\right)$. Now suppose that $\alpha$ is a nonlimit ordinal, say $\alpha=\delta+1$. Define $M_{\delta}$ as in 3.3. Then $\left|M_{\delta}\right| \leqslant \omega$. Let $\left\{a_{\delta i} \mid i \in \omega\right\}$ be an enumeration of $M_{\delta}$. Then for each $i \in \omega$ the set $T \backslash\left\{a_{\delta i}\right\}$ has at most countably many components and consequently has at most countably many components not intersecting $T_{\delta}$. Choose an endpoint $b_{\delta i j}$ from the $j$ th component disjoint from $T_{\delta}$ of $T \backslash\left\{a_{\delta i}\right\}$. We put

$$
T_{\alpha}:=T_{\delta} \cup \bigcup_{\substack{i \in \omega \\ j \in \omega}} S\left(a_{\delta i}, b_{\delta i j}\right)
$$


First we claim that $T_{\alpha}$ is closed in $T$ (it is obvious that $T_{\alpha}$ is connected). Take $x \notin T_{\alpha}$. Then $r_{\delta}(x)=a_{\delta i}$ for some $i \in \omega$; in addition $x \in C_{i j}$ for some component $C_{i j}$ of $T \backslash\left\{a_{\delta i}\right\}$. Let $b_{\delta i j}$ be the endpoint chosen from this component. Since $r_{\delta}: T \rightarrow T_{\delta}$ maps $C_{i j}$ onto $a_{\delta i}$ we see that

$$
U:=C_{i j} \backslash S\left(a_{\delta i}, b_{\delta i j}\right)
$$

is an open neighbourhood of $x$ disjoint from $T_{\alpha}$. We conclude that $T_{\alpha}$ is a metrizable subcontinuum of $T$ since clearly $T$ is separable. In addition the point $x$ cannot be an element of $r_{\alpha}^{-1}(y)$ for some $y \in T_{\delta} \subset T_{\alpha}$, where $r_{\alpha}$ is the canonical retraction of $T$ onto $T_{\alpha}$. For suppose to the contrary that there were such a $y$. Since $r_{\alpha}\left[\left\{a_{\delta i}\right\} \cup C_{i j}\right]=S\left(a_{\delta i}, b_{\delta i j}\right)$ the point $y$ equals $a_{\delta i}$. Then $S\left(x, a_{\delta i}\right) \cap S\left(a_{\delta i}, b_{\delta i j}\right)=\left\{a_{\delta i}\right\}$ which implies that $a_{\delta i} \in S\left(x, b_{\delta i j}\right)$; in other words $a_{\delta i}$ separates $x$ from $b_{\delta i j}$. Then $x$ and $b_{\delta i j}$ are not in the same component of $T \backslash\left\{a_{\delta i}\right\}$, which is a contradiction.

If $\alpha$ is a limit ordinal then we put $T_{\alpha}:=\operatorname{cl}_{T}\left(\cup_{\beta<\alpha} T_{\beta}\right)$. Then $T_{\alpha}$ is metrizable since $\alpha$ is a countable ordinal. Since $T$ is not separable for all $\beta<\alpha$ we have that $T_{\beta} \subsetneq T_{\alpha}$. This completes the transfinite construction.

We claim that $\cup_{\alpha<\omega_{1}} T_{\alpha}=T$. Indeed, assume to the contrary there exists an $x \in T \backslash \cup_{\alpha<\omega_{1}} T_{\alpha}$. First of all, notice that since $T$ is first countable (cf. Theorem 3.1) the set $Z:=\cup_{\alpha<\omega_{1}} T_{\alpha}$ is closed in $T$ and hence is a proper subcontinuum of $T$. Let $r: T \rightarrow Z$ be the canonical retraction. Suppose that $r(x) \in T_{\alpha}\left(\alpha<\omega_{1}\right)$. By construction of the retractions of Proposition 2.2 we see that

$$
r(x)=r_{\alpha}(x)=r_{\alpha+1}(x),
$$

which contradicts (ii) since $r_{\alpha}(x) \in T_{\alpha}$ and $x \in r_{\alpha+1}^{-1}\left(r_{\alpha}(x)\right) \backslash\left\{r_{\alpha}(x)\right\}$.

For every $\alpha<\beta<\omega_{1}$ define $r_{\beta \alpha}: T_{\beta} \rightarrow T_{\alpha}$ by $r_{\beta \alpha}:=r_{\alpha} \uparrow T_{\beta}$. Then, by Lemma 3.3(i) for each $\alpha<\beta$ the diagram

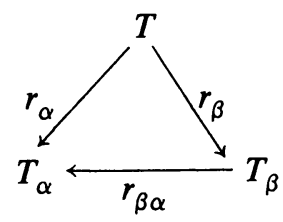

commutes. It follows that $\left(T_{\alpha}, r_{\alpha \beta}, \omega_{1}\right)$ is an inverse system such that

$$
T \approx \operatorname{inv} \lim \left(T_{\alpha}, r_{\alpha \beta}, \omega_{1}\right) ;
$$

for take distinct $x, y \in T$. Then since $T=\cup_{\alpha<\omega_{1}} T_{\alpha}$ there is an $\alpha<\omega_{1}$ such that $x$ and $y$ both belong to $T_{\alpha}$, hence $r_{\alpha}(x) \neq r_{\alpha}(y)$ and consequently the mapping $e: T \rightarrow \operatorname{inv} \lim \left(T_{\alpha}, r_{\alpha \beta}, \omega_{1}\right)$ defined by $e(x)_{\alpha}=r_{\alpha}(x)$ is a homeomorphism.

Next we construct for every $\alpha<\omega_{1}$ a continuous surjection $\xi_{\alpha}: I \rightarrow T_{\alpha}$ and for each $\alpha<\beta<\omega_{1}$ a monotone surjection $f_{\alpha \beta}: I \rightarrow I$ such that the following diagram commutes 


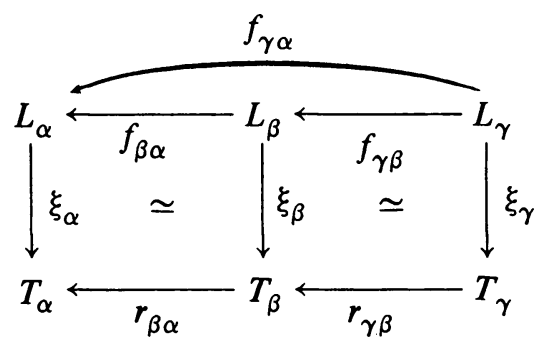

For the sake of convenience we consider a collection of $\omega_{1}$ different copies of $I$ called $\left\{L_{\alpha} \mid \alpha<\omega_{1}\right\}$. In addition, we construct the mappings $\xi_{\alpha}$ in such a way that $\xi_{\alpha}$ is two-to-one in all but countably many points for every nonlimit ordinal $\alpha<\omega_{1}$.

Suppose that the construction is completed for all $\alpha<\gamma$. If $\gamma=0$ then let $L_{0}:=[0,1]$. Let $\phi:\left[0, \frac{1}{2}\right] \rightarrow S\left(a_{0}, b_{0}\right)=T_{0}$ be an (order) isomorphism. Then define $\xi_{0}: L_{0} \rightarrow T_{0}$ by $\xi_{0}(x):=\phi(\min \{x, 1-x\})$. Next suppose that $\gamma=\delta+$ 1. If $M_{\delta}$ is dense in itself (cf. Lemma 3.3) then we divide the $M_{\delta}$ into two subsets $M_{\delta}^{+}$and $M_{\delta}^{-}$which are dense in $M_{\delta}$ (notice that $M_{\delta}$ is homeomorphic to the space of the rationals). Let $a_{\delta i} \in M_{\delta}^{+}$; now let $v_{i}$ be the first member of $L_{\delta}$ such that $\xi_{\delta}\left(v_{i}\right)=a_{\delta i}$. If $a_{\delta i} \in M_{\delta}^{-}$or if $M_{\delta}$ is not dense in itself then we choose $v_{i}$ to be the last member of $L_{\delta}$ such that $\xi_{\delta}\left(v_{i}\right)=a_{\delta i}$. For each segment $S\left(a_{\delta i}, b_{\delta i j}\right)$ there exists a mapping $\xi_{\delta i j}$ from a copy $L_{\delta i j}$ of $I$ onto $S\left(a_{\delta i}, b_{\delta i j}\right)$ such that $\xi_{\delta i j}(0)=\xi_{\delta i j}(1)=a_{\delta i}$ and which is two-to-one except on $b_{\delta i j}$ and which in addition is an isomorphism with respect to the cutset ordering on both $\left[0, \frac{1}{2}\right]$ and $\left[\frac{1}{2}, 1\right]$.

We define

$$
L_{\gamma}:=L_{\delta} \cup \bigcup\left\{L_{\delta i j} \backslash\left\{1_{\delta i j}\right\} \mid i, j \in \omega\right\}
$$

and we obtain an ordering on it by defining:

$$
\left\{\begin{array}{l}
\text { if } x, y \in L_{\delta} \text { and } x<y \text { in } L_{\delta} \text { then also in } L_{\gamma} ; \\
\text { if } x \in L_{\delta} \text { and } y \in L_{\delta i j} \text { and } x<v_{i} \text { in } L_{\delta} \text { then } x<y \text { in } L_{\gamma} ; \\
\quad \text { if } x \geqslant v_{i} \text { in } L_{\delta} \text { then } x>y \text { in } L_{\gamma} ; \\
\text { if } x \in L_{\delta i j} \text { and } y \in L_{\delta m n} \text { and } v_{i}<v_{m} \text { in } L_{\delta} \text { then } x<y \text { in } L_{\gamma} ; \\
\text { if } x \in L_{\delta i j} \text { and } y \in L_{\delta i k} \text { and } j<k \text { then } x<y \text { in } L_{\gamma} ; \\
\text { if } x, y \in L_{\delta i j} \text { and } x<y \text { in } L_{\delta i j} \text { then } x<y \text { in } L_{\gamma} .
\end{array}\right.
$$

The mapping $\xi_{\gamma}$ is defined to be $\xi_{\delta}$ on $L_{\delta}$ and $\xi_{\delta i j}$ on $L_{\delta i j} \backslash\left\{1_{\delta i j}\right\}$; in addition $f_{\gamma \delta}$ is the identity on $L_{\delta}$ and $\left\{v_{i}\right\}$ on $L_{\delta i j} \backslash\left\{1_{\delta i j}\right\}$. For all $\alpha<\delta$ the mapping $f_{\gamma \alpha}$ is defined to be the composition $f_{\delta \alpha} \circ f_{\gamma \delta}$.

In this way we can consider $L_{\gamma}$ to be constructed from $L_{\delta}$ by replacing countably many times a point by a closed unit interval, and in this way it is easy to show that the $L_{\gamma}$ is homeomorphic to the closed unit interval and $f_{\gamma \delta}$ is 
a monotone mapping (cf. Lemma 4.1). The continuity of $\xi_{\gamma}$ can be checked directly if we consider the inverse images of components of points in $T_{\gamma}$ (the components of points in $T_{\gamma}$ form an open subbase for $T_{\gamma}$, as can easily be seen).

If $\gamma$ is a limit ordinal then we take $L_{\gamma}:=\operatorname{inv} \lim \left(L_{\alpha}, f_{\alpha \beta}, \gamma\right)$. Then since $\gamma$ is a countable ordinal, $L_{\gamma}$ is again homeomorphic to the closed unit interval and the mappings $f_{\gamma \alpha}$ and $\xi_{\gamma}$ can be defined in the natural way (because $T_{\gamma} \approx \operatorname{inv} \lim \left(T_{\alpha}, r_{\alpha \beta}, \gamma\right)$ which can be proved in practically the same way as $T \approx \operatorname{inv} \lim \left(T_{\alpha}, r_{\alpha \beta}, \omega_{1}\right)$ above). This completes the transfinite construction.

Let $L$ be inv $\lim \left(L_{\alpha}, f_{\alpha \beta}, \omega_{1}\right)$ with projections $\left\{f_{\alpha}: L \rightarrow L_{\alpha} \mid \alpha<\omega_{1}\right\}$. Let $\xi$ : $L \rightarrow \operatorname{inv} \lim \left(T_{\alpha}, r_{\alpha \beta}, \omega_{1}\right) \approx T$ be defined by $\xi(x)_{\alpha}=\xi_{\alpha}\left(f_{\alpha}(x)\right)$. Then $\xi$ is a continuous surjection. Note that $L$ is an ordered compactum (cf. Capel [1]) since the mappings $f_{\alpha \beta}$ are monotone $\left(\beta<\alpha<\omega_{1}\right)$. Since $T$ is not separable, the space $L$ cannot be separable either, and the only thing left to prove is that $L$ satisfies the countable chain condition.

The space $T_{\delta+1} \backslash T_{\delta}$ is a countable union of half open intervals. If $M_{\delta+1}$ is dense in $T_{\delta+1} \backslash T_{\delta}$ (notice that by construction $M_{\delta+1} \subset T_{\delta+1} \backslash T_{\delta}$ !) then $M_{\delta+1}$ is dense in itself and hence can be divided into two dense subsets. If $M_{\delta+1}$ is not dense then there is a nonvoid open set $O_{\delta+1}$ (open in $T_{\delta+1} \backslash T_{\delta}$ ) in $T_{\delta+1} \backslash T_{\delta}$ disjoint from $M_{\delta+1}$. Clearly $O_{\delta+1}$ is also open in $T_{\delta+1}$ since $T_{\delta}$ is closed in $T_{\delta+1}$. Moreover, since $r_{\delta+1}$ maps $T \backslash T_{\delta+1}$ onto $M_{\delta+1}$ we find that $r_{\delta+1}^{-1}\left[O_{\delta+1}\right]=O_{\delta+1}$ and consequently $O_{\delta+1}$ is open in $T$ too. From this observation it follows that there exist only countably many $\delta$ 's for which $M_{\delta+1}$ is not dense in $T_{\delta+1} \backslash T_{\delta}$ and consequently we can find a $\theta<\omega_{1}$ such that $M_{\delta+1}$ is dense in $T_{\delta+1} \backslash T_{\delta}$ for all $\theta<\delta<\omega_{1}$.

Let $\mathcal{C}$ be a collection of pairwise disjoint nonvoid connected open subsets of $L$. We will prove that $|\mathcal{C}| \leqslant \omega$. For each $C \in \mathcal{C}$ we can define an ordinal number $\phi(C)<\omega_{1}$ as the least number $\alpha<\omega_{1}$ such that int $L_{L_{m}}\left(f_{\alpha}[C]\right) \neq \varnothing$. Since $f_{\alpha}$ is monotone (cf. Capel [1]) we have that for all $\alpha \geqslant \phi(C)$ the set

$$
C^{*}=f_{\alpha}^{-1}\left[\operatorname{int}_{L_{\alpha}}\left(f_{\alpha}[C]\right)\right]
$$

is an open interval in $C$. The number $\phi(C)=\alpha$ can never be a limit ordinal since for limit ordinals $\beta$ we have that $L_{\beta}=\operatorname{inv} \lim \left(L_{\gamma}, f_{\gamma \delta}, \beta\right)$ and

$$
\left\{f_{\beta \gamma}^{-1}[O] \mid O \text { is open in } L_{\gamma}(\gamma<\beta)\right\}
$$

is a base for $L_{\beta}$. If $\alpha \leqslant \theta$, where $\theta$ is as defined above, then

$$
f_{\theta}\left[C^{*}\right]=f_{\theta \alpha}^{-1}\left[\operatorname{int}_{L \alpha}\left(f_{\alpha}[C]\right)\right]
$$

is an open set in $L_{\theta}$ and $C^{*}=f_{\theta}^{-1}\left[f_{\theta}\left[C^{*}\right]\right]$. If $\alpha_{1}=\phi\left(C_{1}\right) \leqslant \theta$ and $\alpha_{2}=\phi\left(C_{2}\right)$ $\leqslant \theta$ then $f_{\theta}\left[C_{1}^{*}\right] \cap f_{\theta}\left[C_{2}^{*}\right]=\varnothing$ since $C_{1} \cap C_{2}=\varnothing$. Since $L_{\theta}$ is ccc it follows that $\phi(C) \leqslant \theta$ for at most countably many $C \in \mathcal{C}$ and hence we can restrict our attention to the collection

$$
\mathscr{D}:=\left\{f_{\alpha}^{-1}\left[\operatorname{int}_{L_{\alpha}}\left(f_{\alpha}[C]\right)\right] \mid C \in \mathcal{C} \text { and } \alpha=\phi(C)>\theta\right\} .
$$


Fix $D \in \mathscr{D}$, say $D=f_{\alpha}^{-1}\left[\operatorname{int}_{L_{\alpha}}\left(f_{\alpha}[C]\right)\right]$ where $C \in \mathcal{C}$ and $\alpha=\phi(C)$. Let $\delta$ be the predecessor of $\alpha$. Define $D_{\alpha}:=f_{\alpha}[D]$. Then $D_{\alpha}$ is an open interval in $L_{\alpha}$ but $f_{\alpha \delta}\left[D_{\alpha}\right]$ is convex without interior in $L_{\delta}$, therefore $f_{\alpha \delta}\left[D_{\alpha}\right]$ has to be some point $v_{i}$ of $L_{\delta}$ with $\xi_{\delta}\left(v_{i}\right)=a_{\delta i}(i \in \omega)$. Therefore $D_{\alpha}$ must be contained in

$$
\cup\left\{L_{\delta i j} \backslash\left\{1_{\delta i j}\right\} \mid j \in \omega\right\}
$$

and consequently there exists an open interval $\left(l_{0}, l_{1}\right)$ in some $L_{\delta i j}$ which is entirely contained in $D_{\alpha}$. Without loss of generality we may assume that either $l_{0}$ and $l_{1}$ are both smaller than $\frac{1}{2}$ or both larger than $\frac{1}{2}$. Assume that $l_{0}<l_{1}<\frac{1}{2}$. The set $\left(l_{0}, l_{1}\right)$ is mapped by $\xi_{\alpha}$ onto the open interval $\left(t_{0}, t_{1}\right)$ in the set $S\left(a_{\delta \mathrm{i}}, b_{\delta i j}\right)$ in $T$. Since $\left(t_{0}, t_{1}\right)$ is open in $T_{\alpha} \backslash T_{\delta}$ and $M_{\alpha}^{+}$is dense in $T_{\alpha} \backslash T_{\delta}$ there is a point $a_{\alpha k} \in M_{\alpha}^{+} \cap\left(t_{0}, t_{1}\right)$. In the construction of $L_{\alpha+1}$ we have assigned a point $v_{k}$ to $a_{\alpha k}$ and since $a_{\alpha k} \in M_{\alpha}^{+}$and $l_{0}<l_{1}<\frac{1}{2}$ the point $v_{k}$ is in $\left(l_{0}, l_{1}\right)$. Let $E_{D} \subset T$ be defined by

$$
E_{D}:=r_{\alpha}^{-1}\left[\left\{a_{\alpha k}\right\}\right] \backslash\left\{a_{\alpha k}\right\} \text {. }
$$

By Lemma 3.3(ii) this set is open in $T$ and

$$
r_{\alpha+1}\left[E_{D}\right]=\bigcup_{j \in \omega} S\left(a_{\alpha k}, b_{\alpha k j}\right) \backslash\left\{a_{\alpha k}\right\}
$$

and consequently

$$
\xi_{\alpha+1}^{-1}\left[r_{\alpha+1}\left[E_{D}\right]\right] \subset \bigcup_{j \in \omega} L_{\alpha k j}
$$

and therefore $f_{\alpha+1, \alpha}\left[\xi_{\alpha+1}^{-1}\left[r_{\alpha+1}\left[E_{D}\right]\right]\right]=\left\{v_{k}\right\}$. We obtain

$$
\xi^{-1}\left[E_{D}\right] \subset f_{\alpha+1}^{-1}\left[\bigcup_{j \in \omega} L_{\alpha k j}\right] \subset f_{\alpha}^{-1}\left(v_{k}\right) \subset D .
$$

If we assume that $\frac{1}{2}<l_{0}<l_{1}$ we can follow the same procedure for some $a_{\alpha k}$ in $M_{\alpha}^{-} \cap \xi_{\alpha}\left[\left(l_{0}, l_{1}\right)\right]$ and also in this case we find an open subset $E_{D}$ of $T$ such that $\xi^{-1}\left[E_{D}\right] \subset D$. Since the collection $\mathscr{D}$ is pairwise disjoint, the corresponding collection $\mathcal{E}=\left\{E_{D} \mid D \in \mathscr{D}\right\}$ is also pairwise disjoint. The space $T$ satisfies the countable chain condition and therefore neither $\mathcal{E}$ nor $\mathscr{D}$ can be uncountable. This finishes the proof.

5.2 COROllary. The existence of a Souslin line is equivalent to the existence of a Souslin dendron.

\section{REFERENCES}

1. C. E. Capel, Inverse limit spaces, Duke Math. J. 21 (1954), 233-245.

2. J. L. Cornette, "Image of Hausdorff arc" is cyclically extensible and reducible, Trans. Amer. Math. Soc. 199 (1974), 253-267.

3. M. J. Faber, Metrizability in generalized ordered spaces, MC tract 53, Mathematisch Centrum, Amsterdam, 1975.

4. G. L. Gurin, On tree-like spaces, Vestnik Moskov. Univ. Ser. I Mat. Meh. 24 (1969), no. 1, 9-12. (Russian)

5. R. B. Jensen, The fine structure of the constructible universe, Ann. Math. Logic 4 (1972), 229-306. 
6. I. Juhász, Martin's axiom solves Ponomarev's problem, Bull. Acad. Polon. Sci. Sér. Sci. Math. Astronom. Phys. 18 (1970), 71-74.

7. H. Kok, Connected orderable spaces, MC tract 49, Mathematisch Centrum, Amsterdam, 1974.

8. J. van Mill and A. Schrijver, Subbase characterizations of compact topological spaces (to appear).

9. J. van Mill and M. van de Vel, Subbases, convex sets and hyperspaces (to appear).

10. V. V. Proizvolov, On peripherally bicompact tree-like spaces, Soviet Math. Dokl. 10 (1969), 1491-1493.

11. On the hereditary and collective normality of a peripherally compact tree-like space, Soviet Math. Dokl. 11 (1970), 1072-1075.

12. M. E. Rudin, Souslin's conjecture, Amer. Math. Monthly 76 (1969), 1113-1119.

13. Lectures on set theoretic topology, CBMS Regional Conf. Ser. in Math., no. 23, Amer. Math. Soc., Providence, R. I., 1975, pp. 1-76.

14. L. E. Ward, Topology, Dekker, New York, 1972.

Department of Mathematics, Free University, De Boelelaan 1081, Postbus 7161, AMSTERDAM, THE NETHERLANDS 